\title{
Strength prediction of notched thin ply laminates using finite fracture mechanics and the phase field approach
}

\author{
J. Reinoso ${ }^{\mathrm{a}, *}$, A. Arteiro ${ }^{\mathrm{b}}$, M. Paggi ${ }^{\mathrm{c}}$, P.P. Camanho ${ }^{\mathrm{b}, \mathrm{d}}$ \\ ${ }^{a}$ Elasticity and Strength of Materials Group, School of Engineering, Universidad de Sevilla, Camino de los Descubrimientos \\ s/n, 41092, Seville, Spain \\ ${ }^{b}$ DEMec, Faculdade de Engenharia, Universidade do Porto, Rua Dr. Roberto Frias, 4200-465 Porto, Portugal \\ ${ }^{c}$ IMT School for Advanced Studies Lucca, Piazza San Francesco 19, 55100 Lucca, Italy \\ ${ }^{d}$ INEGI, Instituto de Ciência e Inovação em Engenharia Mecânica e Engenharia Industrial, Rua Dr. Roberto Frias, 400, \\ 4200-465 Porto, Portugal
}

\begin{abstract}
Thin ply laminates are a new class of composite materials with great potential for application in the design of thinner and highly optimized components, resulting in potential weight savings and improved mechanical performance. These new composites can stir the development of lighter structures, overcoming current design limitations as well as notably reducing the onset and development of matrix cracking and delamination events. This paper presents the application of two recent modeling methods for the failure analysis and strength prediction of open-hole thin ply laminates under tensile loading, which exhibit a brittle response upon failure: (i) the analytical coupled energy-stress Finite Fracture Mechanics (FFMs) technique, and (ii) the FE-based Phase Field (PF) approach for fracture that is incorporated into an enhanced assumed solid shell element. The predictions obtained using both strategies are compared with experimental data. These correlations exhibit a very satisfactory level of agreement, proving the robustness and reliability of both methods under consideration.
\end{abstract}

Keywords: A. Composite materials; B. FE-modeling; C. Damage modeling; D. Shells.

\section{Introduction}

Thin ply laminates, composed by flat and straight plies, with dry ply thicknesses as low as $0.015 \mathrm{~mm}$, are a new class of advanced composite materials with potential benefits that can make composite laminates and structures thinner and, consequently, lighter. For a given laminate thickness, the use of thin plies allow more fiber orientation angles to be accommodated, which is particularly interesting for thin laminates; therefore the design space can be substantially widened. This includes the possibility of using smaller relative fiber orientations between adjacent plies, which is beneficial to mitigate the initiation and propagation of interlaminar fracture events between adjacent plies [1]. The incorporation of thin plies also motivates the

\footnotetext{
* Corresponding authors

Email address: jreinoso@us.es (J. Reinoso)
} 
integration of simpler lay-up rules, such as laminate asymmetry and more flexible ply drops due to easier laminate homogenization, which can contribute to notable reductions in production times.

By reducing the ply thickness in a multidirectional laminate, the in situ effect, which is characterized by an increase in transverse strength of a lamina constrained between two plies with different fiber orientation angles, gains additional importance $[2,3]$. Matrix cracking and delamination can, therefore, be substantially reduced $[4,5,6,7,8,9,10]$ without the use of special resins and/or through-the-thickness reinforcements, providing high strength, enhancing fatigue life, and improving resistance to leakage events.

Apart from the previous characteristics, thin plies also exhibit more uniform fiber orientations and distributions [9], which can potentially reduce the presence of weak zones within the specimen. A direct consequence of the very uniform microstructure of thin plies is an improved unidirectional fiber compressive strength [9]. Moreover, there is also potential for enhanced textile and non-crimp fabric reinforcements, whose handling and lay-up procedures, even over complex shapes, are much easier to be performed. Thus, thinner fiber tows can result in lower crimp angles and in a reduced impact of the stitching yarns.

With such potential benefits from both mechanical and design points of view, it is important to understand how to efficiently and accurately model the mechanical response of thin ply laminates, in particular in the presence of stress concentrations. In fact, structural components typically include geometrical discontinuities for access or assembly purposes or they are susceptible to discrete through-penetration in-service damage, which result in severe stress concentrations. Particularly, such stress concentrations are responsible for the onset and growth of discrete damage in the composite structure, which needs to be accurately modeled to ensure reliable strength predictions.

In the related literature, different damage models formulated at the composite ply level have been proposed in the last years $[11,12,13,14,15,16,17,18]$. However, for laminates with very thin plies, numerical modeling at the ply level turns into a rather impractical approach from an operative standpoint. In fact, compared with conventional ply grades, for the same laminate thickness, thin ply laminates have to accommodate a significantly larger number of plies. If each ply needs to be represented individually for application of a damage model at the ply level, the required discretization and the size of the finite element model will rapidly become unsuitable for application, at least at an industrial level. But the possibility to accommodate more plies also means that macro-mechanical homogenization is much easier to achieve. Moreover, thin ply laminates develop notably lower interlaminar stresses, resulting in a recognized delamination suppression capability $[4,6,7,8,9,10]$. They also have considerably higher in situ strengths $[2$, $3]$, precluding matrix cracking before ultimate laminate failure $[4,5,6,7,8,10]$. Therefore, their mechanical behavior is suitable of representation by a homogenized quasi-brittle material, making macroscopic models formulated at the laminate level ideal for the analysis of their mechanical response.

Recently, analytical coupled energy-stress finite fracture mechanics (FFMs) criteria were proposed in order to overcome the limitations of the classical energy-based Griffith framework for fracture predictions 
$[19,20]$. FFMs envisages a coupled failure criterion through the combination of a stress criterion and an energy criterion, i.e. both criteria should be satisfied simultaneously, to predict an abrupt onset of a finiteextension crack $[21,22]$. This approach has been successfully applied to different engineering applications and has shown the ability of accurately estimating the notched response of composite laminates subjected to simple loading scenarios using only independently measured laminate properties [23, 24]. However, for more complex geometries and loading scenarios, such analytical criteria present notable difficulties due to the fact that the computation of closed-form solutions of the specific stress field and the energy release rate is rather complex.

In order to overcome the current limitations of FFMs, the development of computational modeling strategies to simulate such complex scenarios becomes a matter of recurrent investigation. In this context, numerous computational methods using smeared (based on Continuum Damage Mechanics, CDM) [25] and explicit (extended- and enhanced finite element procedures, XFEM and EFEM, respectively) [26, 27] crack representations, peridynamics-based (PD) techniques [28], multi-scaled methods [29, 30], among many others, have been proposed in the last decades. Differing from alternative FE-based modeling tools, recent multi-field variational formulations for brittle fracture (usually known as phase field, PF, methods) have received a great deal of attention due to their accuracy and robustness in a wide range of applications, see $[31,32,33]$ and the references therein. PF methods can be understood as a particular type of gradient enhanced (nonlocal) method for brittle fracture, which incorporates a characteristic length to regularize the localized failure. One of the main advantages of PF models over alternative methods is associated with their diffusive crack modeling, encompassing a spatially smooth continuum formulation. Furthermore, these novel techniques avoid the explicit representation of kinematic discontinuities, and they can be implemented in a straightforward manner for very complex crack patterns. These modeling capabilities are of special relevance in thin walled applications for which, due to their geometric characteristics, the development of numerical methods to simulate crack events has been a matter of intensive research within the last years, see $[34,35,36,37,38]$ and the references therein given.

In light of the previous considerations, this paper is focused on the application of the FFMs and PF methods for the failure analysis and strength prediction of open-hole thin ply composite laminates subjected to tensile loading. On one hand, the FFMs provides a simple and fast theoretical framework, which simultaneously fulfills coupled energy- and stress-based failure criteria. On the other hand, with the aim of providing a more flexible and reliable method for the failure analysis of thin ply laminates under general loading conditions and geometries, the recent PF approach of brittle fracture is employed. This method is especially suitable for triggering brittle fracture events in solids, which are in line with the typical fracture behavior observed in this new type of composite materials $[4,6,7,8,9]$. In the current investigation, the $\mathrm{PF}$ approach is incorporated into a general nonlinear enhanced assumed solid shell formulation due to the slender character of thin ply laminates [39]. Specifically, the current investigation addresses the following 
innovative aspects. First, the PF approach of fracture is originally applied to strength prediction of thin ply composite specimens, adopting an homogenized response at laminate level. Second, the estimations obtained using both FFMs and PF methodologies are compared by first time with the experimental data related to unidirectional ply laminates and bi-angle non-crimp fabrics, validating both methods. The excellent agreement between FFMs and PF approach of fracture and with respect to the experimental data fosters a new paradigm concerning the simulation of fracture phenomena of thin-ply laminates for practical applications. In this concern, the complementarity between both predictive techniques can undoubtedly contribute for the achievement of a higher leave of exploitation of the actual load bearing capabilities of these new materials.

The organization of the paper is as follows. The central aspects of the FFMs and PF approach of brittle fracture are outlined in Section 2. In Section 3, the strength predictions of open-hole thin ply composite laminates using both methods and their corresponding correlation with the experimental data are presented. Finally, the main conclusions are drawn in Section 4.

\section{Modeling strategies: finite fracture mechanics and phase field approach of brittle fracture}

This section outlines the modeling strategies used for the failure prediction of thin ply laminates under tensile loading. Section 2.1 briefly addresses the principal aspects of the analytical method based on the FFMs for its application to composite open-hole specimens. Subsequently, on the computational side, the central derivations with regard to the PF approach of brittle fracture for shell structures, which relies on an enhanced assumed solid shell parametrization, are presented in Section 2.2.

\subsection{Finite Fracture Mechanics}

The development of reliable prediction tools for composite specimens with stress concentrations has been a recurrent target in the last years due to its practical importance. In [23], a coupled FFMs model was proposed to predict the open-hole tensile strength of composite laminates, see also [19, 20]. According to this model, in a two dimensional setting, failure is the result of the propagation of kinematically admissible cracks with finite sizes. In an open-hole specimen subjected to a remote tensile stress parallel to the $x$-axis (Fig. 1), failure occurs when the following system of equations is satisfied:

$$
\left\{\begin{array}{l}
\frac{1}{l_{c}} \int_{R}^{R+l_{c}} \sigma_{x x}(0, y) \mathrm{d} y=X_{T}^{L} \\
\frac{1}{l_{c}} \int_{R}^{R+l_{c}} \mathcal{G}_{I}(a) \mathrm{d} a=\mathcal{G}_{I c}
\end{array}\right.
$$

where $R$ is the hole radius, $\sigma_{x x}(0, y)$ is the stress distribution along the $y$-axis, which is perpendicular to the loading direction, $X_{T}^{L}$ is the laminate un-notched strength, $\mathcal{G}_{I}(a)$ is the mode I energy release rate for a plate with a central circular hole and two symmetric cracks emanating from the hole edge, $\mathcal{G}_{I c}$ is the 
laminate fracture toughness, and $l_{c}$ is the crack extension at failure. According to the system given in Eq. (1), failure occurs when both stress-based and energy-based criteria are simultaneously satisfied. This model is based on an analytical solution, and no empirical adjusting parameters are required, but only the ply elastic properties, the laminate un-notched strength, $X_{T}^{L}$, and the laminate fracture toughness, $\mathcal{G}_{I c}$.

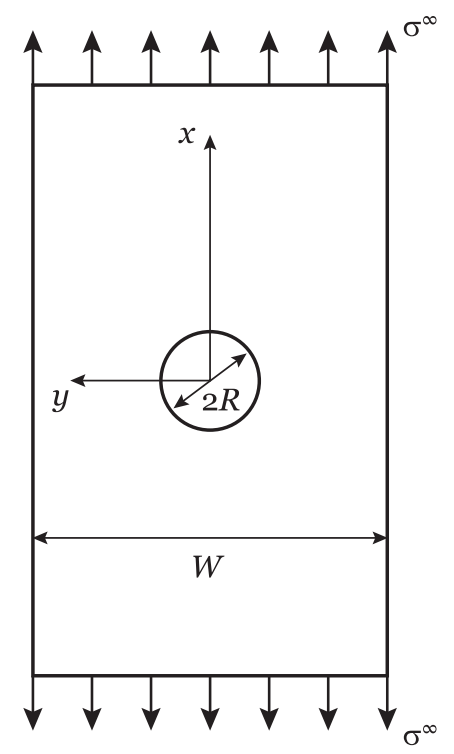

Figure 1: Notched plate with central circular hole.

For the applications under consideration in the current investigation, the stress distribution along the ligament section perpendicular to the loading direction (along the $y$-axis, see Fig. 1 ), $\sigma_{x x}(0, y)$, can be obtained as [41]:

$$
\begin{aligned}
\sigma_{x x}(0, y)= & \frac{K_{T}}{K_{T}^{\infty}} \frac{\sigma^{\infty}}{2}\left[2+\left(\frac{R}{y}\right)^{2}+3\left(\frac{R}{y}\right)^{4}-\right. \\
& \left.-\left(K_{T}^{\infty}-3\right)\left[5\left(\frac{R}{y}\right)^{6}-7\left(\frac{R}{y}\right)^{8}\right]\right], y>R
\end{aligned}
$$

where $K_{T}^{\infty}$ denotes the stress concentration factor at the edge of the hole of an infinite plate [41]:

$$
K_{T}^{\infty}=1+\sqrt{2\left(\sqrt{\frac{E_{x}}{E_{y}}}-\nu_{x y}\right)+\frac{E_{x}}{G_{x y}}}
$$

and $K_{T} / K_{T}^{\infty}$ is the finite-width correction factor, which is accurate for hole diameter-to-width ratios $2 R / W<0.6[41]:$ 


$$
\frac{K_{T}}{K_{T}^{\infty}}=\left[\frac{3(1-2 R / W)}{2+(1-2 R / W)^{3}}+\frac{1}{2}\left(\frac{2 R}{W} M\right)^{6}\left(K_{T}^{\infty}-3\right)\left[1-\left(\frac{2 R}{W} M\right)^{2}\right]\right]^{-1}
$$

In Eq. (3), $E_{x}, E_{y}, G_{x y}$ and $\nu_{x y}$ are the laminate elastic constants; the parameter $M$ in Eq. (4) is calculated as proposed in [41]:

$$
M^{2}=\frac{\sqrt{1-8\left[\frac{3(1-2 R / W)}{2+(1-2 R / W)^{3}}-1\right]}-1}{2(2 R / W)^{2}}
$$

The mode I energy release rate, $\mathcal{G}_{I}(a)$, is calculated as:

$$
\mathcal{G}_{I}(a)=\frac{\left(\mathcal{K}_{I}(a)\right)^{2}}{E}
$$

where $\mathcal{K}_{I}(a)$ is the mode I stress intensity factor and $E$ is the equivalent modulus of the laminate:

$$
\dot{E}=\left(\frac{1+\rho}{2 E_{x} E_{y}}\right)^{-1 / 2}\left(\frac{E_{y}}{E_{x}}\right)^{-1 / 4}
$$

with:

$$
\rho=\frac{\left(E_{x} E_{y}\right)^{1 / 2}}{2 G_{x y}}-\left(\nu_{x y} \nu_{y x}\right)^{1 / 2}
$$

For a plate with a central circular hole of radius $R$ and two symmetric cracks emanating from the hole edge, $\mathcal{K}_{I}(a)$ is given, for an isotropic plate, as [42]:

$$
\mathcal{K}_{I}(a)=\sigma^{\infty} F_{h} F_{w} \sqrt{\pi a}
$$

with:

$$
\begin{gathered}
F_{h}=\sqrt{1-\lambda} f_{n} \\
F_{w}=\sqrt{\sec \left(\frac{\pi R}{W}\right) \sec \left(\frac{\pi a}{W}\right)}
\end{gathered}
$$

where:

$$
f_{n}=1+0.358 \lambda+1.425 \lambda^{2}-1.578 \lambda^{3}+2.156 \lambda^{4}, \quad \lambda=\frac{R}{a}
$$

Using Eqs. (2), (6) and (9) in the system of Eqs. (1), the coupled stress and energy FFMs criterion can be solved for $l_{c}$ and for the remote stress at failure, $\bar{\sigma}^{\infty}$. In $[23,40]$, it was shown that, for laminates exhibiting a fiber-dominated failure mode, the model predictions are reasonably accurate, with improvements over traditional strength prediction methods such as the point-stress and average-stress models [43]. 


\subsection{Phase field approach of brittle fracture}

In order to provide a more versatile tool for modeling brittle fracture of thin ply laminates, this section describes the fundamental ingredients of the PF approach of brittle fracture. This method has been extensively applied to different engineering applications in the last years [31, 32, 33, 44, 45, 46]. One of the main novelties of the present study is the application of this recent nonlocal variational method for brittle fracture, overcoming the limitations of CDM-based approaches and enriched formulations.

\subsubsection{Phase field model for thin-walled structures}

The basic idea of the PF strategy for fracture envisages a smeared crack representation over a zone of width $l$, see the one-dimensional representation in Fig. 2(a). This modeling assumption leads to the definition of the scalar-valued function $\mathfrak{d}$, with $\mathfrak{d}: \mathcal{B}_{0} \times[0, t] \rightarrow[0,1]$, which is a smooth function of $(\mathbf{X}, t)$ in the reference configuration of the body $\mathcal{B}_{0}$, with $\mathfrak{d}=0$ and $\mathfrak{d}=1$ identifying the intact and the cracked states, respectively.

The proposed phase field model used in the current investigation is integrated into a general nonlinear enhanced assumed solid shell formulation in line with the modeling framework outlined in [39], whereby an homogeneized material model is used at laminate level. For an alternative PF definition for shells see also [47]. As mentioned before, this formulation is especially suitable for the analysis of thin ply composite laminates, since the vast majority of such components in aeronautical and aerospace applications are typically conceived as plates or shells.

In this context, the reference shell configuration is denoted by $\mathcal{B}_{0} \subset \mathbb{R}^{3}$, whereas $\mathcal{B}_{t} \subset \mathbb{R}^{3}$ identifies its corresponding current configuration, see Fig. 2(b). The vectors $\mathbf{X}$ and $\mathbf{x}$ stand for the reference and current position vectors, respectively. Along the deformation process, the body undergoes a motion, which is described by the nonlinear operator $\varphi(\mathbf{X}, t): \mathcal{B}_{0} \times[0, t] \rightarrow \mathbb{R}^{3}$, where $[0, t]$ is the time step interval that maps the reference material points $\left(\mathbf{X} \in \mathcal{B}_{0}\right)$ onto the current material points $\left(\mathbf{x} \in \mathcal{B}_{t}\right)$, such that $\mathbf{x}=\varphi(\mathbf{X}, t)$. The displacement-derived deformation gradient is defined as $\mathbf{F}^{u}:=\partial_{\mathbf{X}} \boldsymbol{\varphi}(\mathbf{X}, t)$, where $J^{u}=\operatorname{det}\left[\mathbf{F}^{u}\right]$ is the corresponding Jacobian of the transformation, and $\partial \mathbf{x}$ is the partial derivative with respect to the reference frame.

In line with with the assumptions of the PF modeling [32, 33], the surface integrals defined on sharp crack surfaces can be replaced by volume integrals as follows:

$$
\int_{\Gamma_{c}} \mathcal{G}_{c} \mathrm{~d} \Gamma \approx \int_{\mathcal{B}_{0}} \mathcal{G}_{c} \gamma\left(\mathfrak{d}, \nabla_{\mathbf{X}} \mathfrak{d}\right) \mathrm{d} \Omega,
$$

where $\mathcal{G}_{c}$ is the fracture toughness according to the Griffith fracture theory, and $\gamma\left(\mathfrak{d}, \nabla_{\mathbf{X}} \mathfrak{d}\right)$ identifies the so-called crack surface density functional per unit volume of the solid. A common form for $\gamma\left(\mathfrak{d}, \nabla_{\mathbf{X}} \mathfrak{d}\right)$ is given by the second order Allen-Cahn functional [45]:

$$
\gamma\left(\mathfrak{d}, \nabla_{\mathbf{X}} \mathfrak{d}\right)=\frac{1}{2 l} \mathfrak{d}^{2}+\frac{l}{2}|\nabla \mathbf{X} \mathfrak{d}|^{2},
$$


a

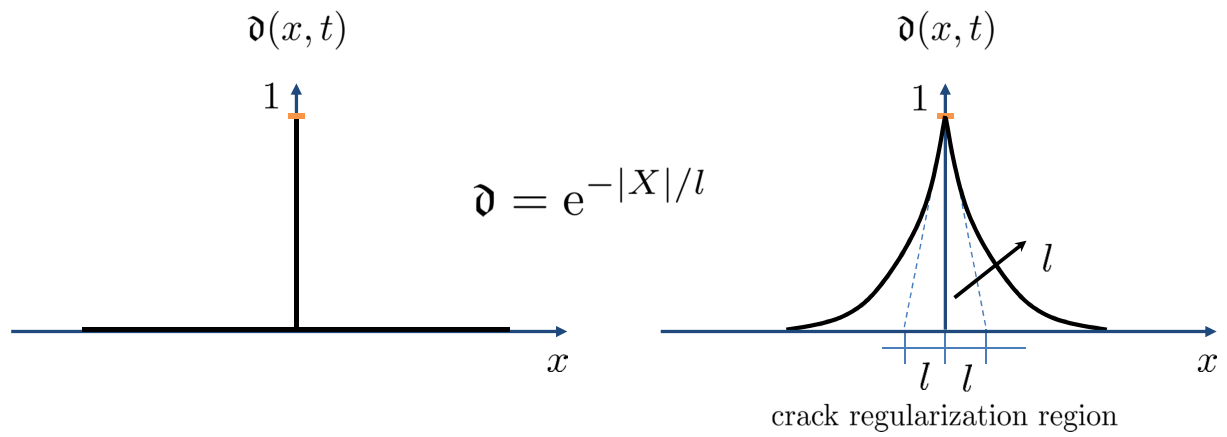

$\mathrm{b}$

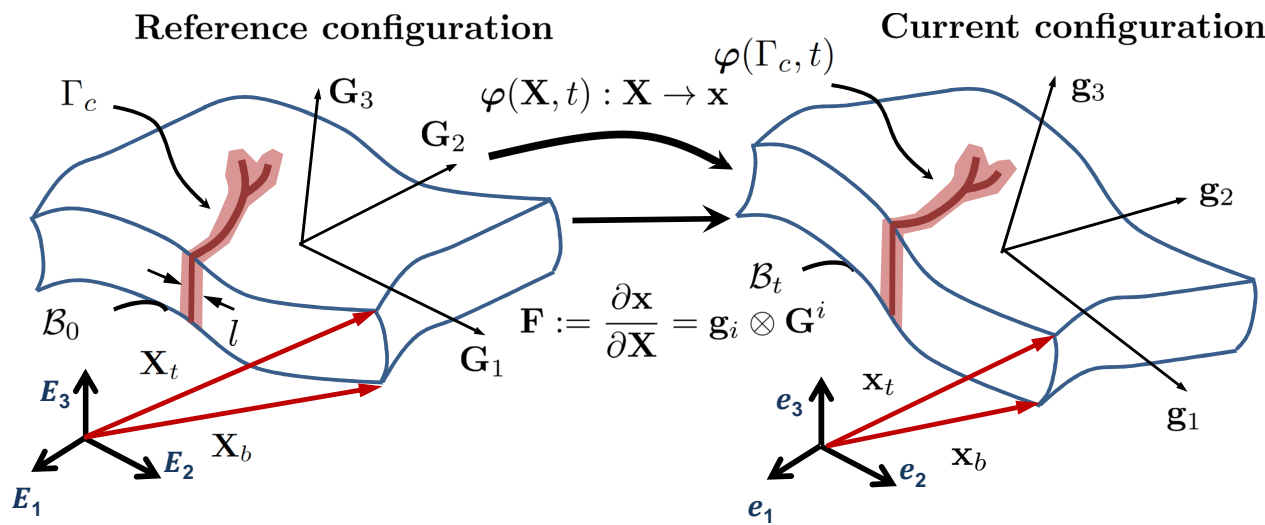

Figure 2: (a) Sharp and diffusive crack representation. (Left) Sharp crack at $x=0$. (Right) Diffusive crack at $x=0$ confining the regularization to the region characterized by the length scale $l$. (b) Definition of the reference and current configurations of the shell body where fracture is considered through the phase field concept on the surface $\Gamma_{c} .\left\{\mathbf{E}_{i}\right\}_{i=1,3},\left\{\mathbf{e}_{i}\right\}_{i=1,3}$ denote the standard Cartesian frame associated with the reference $\mathcal{B}_{0}$ and current $\mathcal{B}_{t}$ configurations, respectively. The co-variant basis in the reference and current configuration respectively read: $\mathbf{G}_{i}(\boldsymbol{\xi}):=\partial \mathbf{X}(\boldsymbol{\xi}) / \partial \xi^{i}, \mathbf{g}_{i}(\boldsymbol{\xi}):=\partial \mathbf{x}(\boldsymbol{\xi}) / \partial \xi^{i}$.

where the characteristic length parameter $l$ controls the regularization band of the model, converging to the sharp crack surface $\Gamma_{c}$ for the limit $l \rightarrow 0$ in the spirit of the so-called $\Gamma$-convergence [31].

Recalling the 6-parameter shell model, an arbitrary thin-walled body can be parameterized according to the solid shell concept [48]. This approach has been extensively used in different engineering applications and has offered several attractive aspects such as the complete avoidance of parametrization of rotational degrees of freedom in nonlinear analysis and the use of unmodified three-dimensional constitutive laws, among other characteristics $[49,50]$. Accordingly, the position vector of any material point, $\mathbf{X}$, can be expressed by points of the top $\mathbf{X}_{t}\left(\xi^{1}, \xi^{2}\right)$ and bottom surfaces $\mathbf{X}_{b}\left(\xi^{1}, \xi^{2}\right)$ of the shell, see Fig. 2(b). This representation can be expressed in the reference and current configurations as:

$$
\begin{aligned}
\mathbf{X}(\boldsymbol{\xi}) & =\frac{1}{2}\left(1+\xi^{3}\right) \mathbf{X}_{t}\left(\xi^{1}, \xi^{2}\right)+\frac{1}{2}\left(1-\xi^{3}\right) \mathbf{X}_{b}\left(\xi^{1}, \xi^{2}\right) . \\
\mathbf{x}(\boldsymbol{\xi}) & =\frac{1}{2}\left(1+\xi^{3}\right) \mathbf{x}_{t}\left(\xi^{1}, \xi^{2}\right)+\frac{1}{2}\left(1-\xi^{3}\right) \mathbf{x}_{b}\left(\xi^{1}, \xi^{2}\right) .
\end{aligned}
$$


The corresponding parametric space is defined as: $\mathcal{A}:=\left\{\boldsymbol{\xi}=\left(\xi^{1}, \xi^{2}, \xi^{3}\right) \in \mathbb{R}^{3} \mid-1 \leq \xi^{i} \leq+1 ; i=\right.$ $1,2,3\}$, where $\left(\xi^{1}, \xi^{2}\right)$ stand for the in-plane directions and $\xi^{3}$ is the thickness direction, with $H$ representing the initial shell thickness.

Based on the previous shell description, the kinematic field adopts the following representation:

$$
\mathbf{u}(\boldsymbol{\xi})=\mathbf{X}(\boldsymbol{\xi})-\mathbf{x}(\boldsymbol{\xi})=\mathbf{v}\left(\xi^{1}, \xi^{2}\right)+\xi^{3} \mathbf{w}\left(\xi^{1}, \xi^{2}\right)
$$

where $\mathbf{v}\left(\xi^{1}, \xi^{2}\right)$ and $\mathbf{w}\left(\xi^{1}, \xi^{2}\right)$ identifies the displacement vectors of the shell midsurface and the director vector, respectively. These vectors can be expressed as

$$
\mathbf{v}\left(\xi^{1}, \xi^{2}\right)=\frac{1}{2}\left[\mathbf{u}_{t}\left(\xi^{1}, \xi^{2}\right)+\mathbf{u}_{b}\left(\xi^{1}, \xi^{2}\right)\right] ; \quad \mathbf{w}\left(\xi^{1}, \xi^{2}\right)=\frac{1}{2}\left[\mathbf{u}_{t}\left(\xi^{1}, \xi^{2}\right)-\mathbf{u}_{b}\left(\xi^{1}, \xi^{2}\right)\right]
$$

where $\mathbf{u}_{t}\left(\xi^{1}, \xi^{2}\right)$ and $\mathbf{u}_{b}\left(\xi^{1}, \xi^{2}\right)$ stand for the displacement vectors of the top and bottom surfaces of the shell, respectively.

The displacement derived Green-Lagrange strain tensor is defined as

$$
\mathbf{E}^{u}:=\frac{1}{2}\left[\left(\mathbf{F}^{u}\right)^{\mathrm{T}} \mathbf{F}^{u}-\mathbb{I}_{2}\right]=\frac{1}{2}\left[g_{i j}-G_{i j}\right] \mathbf{G}^{i} \otimes \mathbf{G}^{j},
$$

where $\mathbb{I}_{2}$ is the material covariant metric. The strain components are arranged as

$$
\mathbf{E}^{u}=\left[E_{11}^{u}, E_{22}^{u}, E_{33}^{u}, 2 E_{12}^{u}, 2 E_{23}^{u}, 2 E_{13}^{u}\right]
$$

It is worth mentioning that the current solid shell model allows a fully $3 \mathrm{D}$ description of the strain field to be simulated, i.e. precluding plane stress assumptions as standard Kirchhoff-Lover or Reissner-Mindlin shell formulations. Moreover, as described in Section 2.2.2, locking pathologies are remedied through the use of a incompatible strain field. Specifically, the 7-parameter shell model according to [51] is recalled through the advocation of the enhanced assumed strain concept, which affects the transverse normal strain component to tackle Poisson thickness locking effects as follows:

$$
E_{33}=E_{33}^{u}+\xi^{3} \tilde{\beta}_{33}\left(\xi^{1}, \xi^{2}\right)
$$

where $\xi^{3} \tilde{\beta}_{33}\left(\xi^{1}, \xi^{2}\right)$ is the linear strain term in the transverse direction.

The previous kinematic ansatz is also adopted for the phase field parametrization within the shell body:

$$
\mathfrak{d}(\boldsymbol{\xi})=\frac{1}{2}\left(1+\xi^{3}\right) \mathfrak{d}_{t}\left(\xi^{1}, \xi^{2}\right)+\frac{1}{2}\left(1-\xi^{3}\right) \mathfrak{d}_{b}\left(\xi^{1}, \xi^{2}\right),
$$

where $\mathfrak{d}_{t}\left(\xi^{1}, \xi^{2}\right)$ and $\mathfrak{d}_{b}\left(\xi^{1}, \xi^{2}\right)$ denote respectively the phase field variables of the top and bottom surfaces of the shell. Correspondingly, this assumption regarding the phase field variable allows a linear approximation over the shell thickness to be performed. This ingredient becomes especially suitable for bending dominated applications where damage states at the top and bottom surfaces are not necessarily uniform so far [39]. 


\subsubsection{Variational and finite element formulations}

In the sequel, the variational and finite element formulations of the developed phase field model for thin-walled composite structures are addressed.

The particular form of the current variational formulation accounts for the consideration of the enhanced assumed strain (EAS) method [52] for the alleviation of Poisson thickness and volumetric locking effects. In particular, the additive decomposition of the Green-Lagrange strain tensor (E) is herein adopted, i.e. $\mathbf{E}=\mathbf{E}^{u}+\tilde{\mathbf{E}}$, where $\mathbf{E}^{u}$ and $\tilde{\mathbf{E}}$ identify the compatible and the incompatible counterparts, respectively [51]. The proposed formulation relies on the exploitation of the multi-field $\mathrm{Hu}$-Washizu variational principle expressed as the stationarity of the following functional of a possibly cracked body:

$$
\Pi(\mathbf{S}, \tilde{\mathbf{E}}, \mathbf{u}, \mathfrak{d})=\int_{\mathcal{B}_{0}} \mathfrak{g}(\mathfrak{d}) \Psi\left(\mathbf{E}^{u}, \tilde{\mathbf{E}}\right) \mathrm{d} \Omega-\int_{\mathcal{B}_{0}} \mathbf{S}: \tilde{\mathbf{E}} \mathrm{d} \Omega+\int_{\mathcal{B}_{0}} \frac{\mathcal{G}_{c} l}{2}\left(\frac{\mathfrak{d}^{2}}{l^{2}}+\left|\nabla_{\mathbf{X}} \mathfrak{d}\right|^{2}\right) \mathrm{d} \Omega+\Pi_{\text {ext }},
$$

where the displacement field $(\mathbf{u})$, the incompatible strain tensor $(\tilde{\mathbf{E}})$, the second Piola-Kirchhoff stress tensor $(\mathbf{S})$ and the phase field variable $(\mathfrak{d})$ are the independent variables, and $\Pi_{\text {ext }}$ denotes the contribution due to the prescribed external actions. In Eq. $(23), \mathfrak{g}(\mathfrak{d})=[1-\mathfrak{d}]^{2}+\mathcal{K}$ is the so-called degradation function, affecting the current stiffness along the loading process, and the parameter $\mathcal{K} \approx 0$ stands for a residual positive parameter that prevents numerical instabilities due to fully degraded material response [32]. It is noted that the degradation function $\mathfrak{g}$ affects the free-energy function, whereas the third integral in Eq. (23) identifies the term associated with the energy dissipation due to fracture. It can be also observed that the variational form encompasses a energy competition between the deformation and the fracture dissipation terms.

Based on standard arguments, the definition of the second Piola-Kirchhoff stress tensor $(\mathbf{S})$ and the driving force of the phase field $(\mathcal{H})$ read:

$$
\mathbf{S}:=\frac{\partial \bar{\Psi}\left(\mathbf{E}^{u}, \tilde{\mathbf{E}}, \mathfrak{d}\right)}{\partial \mathbf{E}} ; \quad \mathcal{H}:=\frac{\partial \bar{\Psi}\left(\mathbf{E}^{u}, \tilde{\mathbf{E}}, \mathfrak{d}\right)}{\partial \mathfrak{d}}, \quad \text { with } \bar{\Psi}\left(\mathbf{E}^{u}, \tilde{\mathbf{E}}, \mathfrak{d}\right)=\mathfrak{g}(\mathfrak{d}) \Psi\left(\mathbf{E}^{u}, \tilde{\mathbf{E}}\right) .
$$

Through the imposition of the orthogonality condition between the interpolation spaces of the stress and the incompatible strain fields, the stress field is eliminated from the variational form given in Eq. (23) [52]. Thus, the residual equations of the corresponding multi-field problem take the form:

$$
\begin{gathered}
\mathcal{R}^{u}(\mathbf{u}, \delta \mathbf{u}, \tilde{\mathbf{E}}, \mathfrak{d})=\mathcal{R}_{\text {int }}^{u}-\mathcal{R}_{\text {ext }}^{u}=\int_{\mathcal{B}_{0}} \mathfrak{g}(\mathfrak{d})\left[\frac{\partial \Psi}{\partial \mathbf{E}}: \frac{\partial \mathbf{E}^{u}}{\partial \mathbf{u}} \delta \mathbf{u}\right] \mathrm{d} \Omega+\delta \Pi_{\text {ext }}(\mathbf{u})=0, \quad \forall \delta \mathbf{u} \in \mathfrak{V}^{u} \\
\mathcal{R}^{\tilde{E}}(\mathbf{u}, \tilde{\mathbf{E}}, \delta \tilde{\mathbf{E}}, \mathfrak{d})=\int_{\mathcal{B}_{0}} \mathfrak{g}(\mathfrak{d})\left[\frac{\partial \Psi}{\partial \mathbf{E}}: \delta \tilde{\mathbf{E}}\right] \mathrm{d} \Omega=0, \quad \forall \delta \tilde{\mathbf{E}} \in \mathfrak{V}^{\tilde{E}} \\
\mathcal{R}^{\mathfrak{d}}(\mathbf{u}, \tilde{\mathbf{E}}, \mathfrak{d}, \delta \mathfrak{d})=\int_{\mathcal{B}_{0}}-2(1-\mathfrak{d}) \delta \mathfrak{d} \Psi\left(\mathbf{E}^{u}, \tilde{\mathbf{E}}\right) \mathrm{d} \Omega+\int_{\mathcal{B}_{0}} \mathcal{G}_{c} l\left[\frac{1}{l^{2}} \mathfrak{d} \delta \mathfrak{d}+\nabla \mathbf{X}^{\mathfrak{d}} \cdot \nabla \mathbf{X}(\delta \mathfrak{d})\right] \mathrm{d} \Omega=0, \quad \forall \delta \mathfrak{d} \in \mathfrak{V}^{\mathfrak{d}},
\end{gathered}
$$

where $\mathfrak{V}^{u}=\left\{\delta \mathbf{u} \in\left[H^{1}\left(\mathcal{B}_{0}\right)\right]: \delta \mathbf{u}=\mathbf{0}\right.$ on $\left.\partial \mathcal{B}_{0, u}\right\}$ denotes the space of admissible displacement variations $(\delta \mathbf{u}), \mathfrak{V}^{\tilde{E}}=\left[L_{2}\left(\mathcal{B}_{0}\right)\right]$ identifies the admissible space of the enhanced strain modes $(\delta \tilde{\mathbf{E}})$, and finally $\mathfrak{V}^{\mathfrak{D}}=$ 
$\left\{\delta \mathfrak{d} \in \mathcal{H}^{1}\left(\mathcal{B}_{0}\right) \mid \delta \mathfrak{d}=0\right.$ on $\left.\Gamma_{c}\right\}$ stands for the space of admissible test functions for the phase field $(\delta \mathfrak{d})$. The residual vectors corresponding to the kinematic field, the incompatible strains and the phase field are respectively denoted as $\mathcal{R}^{u}(\mathbf{u}, \delta \mathbf{u}, \tilde{\mathbf{E}}, \mathfrak{d}), \mathcal{R}^{\tilde{E}}(\mathbf{u}, \tilde{\mathbf{E}}, \delta \tilde{\mathbf{E}}, \mathfrak{d})$ and $\mathcal{R}^{\mathfrak{o}}(\mathbf{u}, \tilde{\mathbf{E}}, \mathfrak{d}, \delta \mathfrak{d})$.

The set of the coupled nonlinear residual equations given in Eqs. (25)-(27) are solved using a standard incremental-iterative Newton-Rahpson scheme, adopting a fully coupled formulation [39].

The finite element discretization of the previous weak forms Eqs. (25)-(27) is performed via the isoparametric concept. The reference and current configurations of the shell are interpolated through standard trilinear shape functions $N^{I}(\mathbf{N}(\boldsymbol{\xi})$ in matrix notation) as:

$$
\mathbf{X}=\sum_{I=1}^{n_{n}} N^{I}(\boldsymbol{\xi}) \mathbf{X}_{I}=\mathbf{N}(\boldsymbol{\xi}) \widetilde{\mathbf{X}} \quad \text { and } \quad \mathbf{x}=\sum_{I=1}^{n_{n}} N^{I}(\boldsymbol{\xi}) \mathbf{x}_{I}=\mathbf{N}(\boldsymbol{\xi}) \widetilde{\mathbf{x}}
$$

where $\mathbf{X}_{I}$ and $\mathbf{x}_{I}$ denote the discrete nodal values and $n_{n}=8$ identifies the number of nodes at the element level. The nodal position vectors are collected into the respective vectors $\widetilde{\mathbf{X}}$ and $\widetilde{\mathbf{x}}$ for both configurations.

Accordingly, the displacement and the phase field variable $(\mathbf{u}, \mathfrak{d})$, their respective variations $(\delta \mathbf{u}, \delta \mathfrak{d})$ and their increments $(\Delta \mathbf{u}, \Delta \mathfrak{d})$ are approximated as follows:

$$
\begin{aligned}
& \mathbf{u} \approx \mathbf{N}(\boldsymbol{\xi}) \mathbf{d} ; \delta \mathbf{u} \approx \mathbf{N}(\boldsymbol{\xi}) \delta \mathbf{d} ; \Delta \mathbf{u} \approx \mathbf{N}(\boldsymbol{\xi}) \Delta \mathbf{d} \\
& \mathfrak{d}=\mathbf{N}(\boldsymbol{\xi}) \overline{\mathfrak{d}} ; \delta \mathfrak{d}=\mathbf{N}(\boldsymbol{\xi}) \delta \overline{\mathfrak{d}} ; \quad \Delta \mathfrak{d}=\mathbf{N}(\boldsymbol{\xi}) \Delta \overline{\mathfrak{d}}
\end{aligned}
$$

where $\mathbf{d}$ and $\overline{\mathfrak{d}}$ identify the vectors containing the nodal displacements and phase field variables at the element level.

The interpolation of the incompatible strains $(\tilde{\mathbf{E}})$ is performed at the element level by means of the operator $\mathbf{M}(\boldsymbol{\xi})$ according to the scheme:

$$
\tilde{\mathbf{E}} \approx \mathbf{M}(\boldsymbol{\xi}) \varsigma, \quad \delta \tilde{\mathbf{E}} \approx \mathbf{M}(\boldsymbol{\xi}) \delta \varsigma, \quad \Delta \tilde{\mathbf{E}} \approx \mathbf{M}(\boldsymbol{\xi}) \Delta \boldsymbol{\varsigma},
$$

where $(\delta \tilde{\mathbf{E}})$ and $(\Delta \tilde{\mathbf{E}})$ stand for the variation and increment of the incompatible strains, respectively. The particular form of the interpolation operator in the local parametric setting $(\tilde{\mathbf{M}}(\boldsymbol{\xi}))$ for the alleviation of Poisson thickness and volumetric locking effects is chosen as:

$$
\tilde{\mathbf{M}}(\boldsymbol{\xi})=\left[\begin{array}{ccccccc}
\xi^{1} & 0 & 0 & 0 & 0 & 0 & 0 \\
0 & \xi^{2} & 0 & 0 & 0 & 0 & 0 \\
0 & 0 & \xi^{3} & \xi^{1} \xi^{3} & \xi^{2} \xi^{3} & 0 & 0 \\
0 & 0 & 0 & 0 & 0 & \xi^{1} & \xi^{2} \\
0 & 0 & 0 & 0 & 0 & 0 & 0 \\
0 & 0 & 0 & 0 & 0 & 0 & 0
\end{array}\right] .
$$


Note that the operator $\tilde{\mathbf{M}}(\boldsymbol{\xi})$ is transformed to the global Cartesian setting complying with the patch test.

This transformation reads:

$$
\tilde{\mathbf{E}}=\left[\frac{\operatorname{det} \mathbf{J}_{0}}{\operatorname{det} \mathbf{J}}\right] \mathbf{T}_{0} \tilde{\mathbf{M}}(\boldsymbol{\xi}) \varsigma=\mathbf{M}(\boldsymbol{\xi}) \boldsymbol{\varsigma},
$$

where $\mathbf{J}=\left[\mathbf{G}_{1}, \mathbf{G}_{2}, \mathbf{G}_{3}\right]$ and $\mathbf{J}_{0}=\left[\mathbf{G}_{1(0)}, \mathbf{G}_{2(0)}, \mathbf{G}_{3(0)}\right]$ stand respectively for the Jacobian and the corresponding evaluation at the element center, while the components of the transformation matrix $\mathbf{T}$ render: $T^{i j}=\mathbf{G}^{i} \cdot \mathbf{G}_{(0)}^{j}[53,54]$.

To integrate the previous formulation into a nonlinear FE solution scheme, the residual equations introduced above, Eqs. (25)-(27), are consistently linearized through the directional derivative concept. The linearized equations can adopt the form:

$$
\begin{aligned}
& \hat{L}\left[\mathbf{R}^{d}\right](\mathbf{d}, \delta \mathbf{d}, \Delta \mathbf{d}, \overline{\mathfrak{d}}, \Delta \overline{\mathfrak{d}}, \varsigma, \Delta \varsigma)=\mathbf{R}^{d}(\mathbf{d}, \delta \mathbf{d}, \overline{\mathfrak{d}}, \varsigma)+\Delta_{\mathbf{d}} \mathbf{R}^{d} \Delta \mathbf{d}+\Delta_{\overline{\mathfrak{d}}} \mathbf{R}^{d} \Delta \overline{\mathfrak{d}}+\Delta_{\varsigma} \mathbf{R}^{d} \Delta \varsigma \\
& \hat{L}\left[\mathbf{R}^{\varsigma}\right](\mathbf{d}, \Delta \mathbf{d}, \overline{\mathfrak{d}}, \Delta \overline{\mathfrak{d}}, \varsigma, \delta \varsigma, \Delta \varsigma)=\mathbf{R}^{\varsigma}(\mathbf{d}, \overline{\mathfrak{d}}, \varsigma, \delta \varsigma)+\Delta_{\mathbf{d}} \mathbf{R}^{\varsigma} \Delta \mathbf{d}+\Delta_{\overline{\mathfrak{d}}} \mathbf{R}^{\varsigma} \Delta \overline{\mathfrak{d}}+\Delta_{\varsigma} \mathbf{R}^{\varsigma} \Delta \varsigma \\
& \hat{L}\left[\mathbf{R}^{\mathfrak{d}}\right](\mathbf{d}, \overline{\mathfrak{d}}, \delta \overline{\mathfrak{d}}, \Delta \overline{\mathfrak{d}}, \varsigma, \delta \varsigma, \Delta \varsigma)=\mathbf{R}^{\mathfrak{d}}(\mathbf{d}, \overline{\mathfrak{d}}, \delta \overline{\mathfrak{d}}, \varsigma)+\Delta_{\mathbf{d}} \mathbf{R}^{\mathfrak{d}} \Delta \mathbf{d}+\Delta_{\overline{\mathfrak{d}}} \mathbf{R}^{\mathfrak{d}} \Delta \overline{\mathfrak{d}}+\Delta_{\varsigma} \mathbf{R}^{\mathfrak{d}} \Delta \varsigma
\end{aligned}
$$

where $\Delta_{*}[\bullet]$ identifies the directional derivative operator with respect to the field $*$.

In line with this procedure, the coupled system of equations in matrix format adopts the following representation:

$$
\left[\begin{array}{lll}
\mathbf{k}_{d d} & \mathbf{k}_{d \mathfrak{d}} & \mathbf{k}_{d \varsigma} \\
\mathbf{k}_{\mathfrak{d} d} & \mathbf{k}_{\mathfrak{d} \mathfrak{d}} & \mathbf{k}_{\mathfrak{d} \varsigma} \\
\mathbf{k}_{\varsigma d} & \mathbf{k}_{\varsigma \mathfrak{d}} & \mathbf{k}_{\varsigma \varsigma}
\end{array}\right]\left[\begin{array}{c}
\Delta \mathbf{d} \\
\Delta \overline{\mathfrak{d}} \\
\Delta \varsigma
\end{array}\right]=\left[\begin{array}{c}
\mathbf{R}_{\mathrm{ext}}^{d} \\
\mathbf{0} \\
\mathbf{0}
\end{array}\right]-\left[\begin{array}{l}
\mathbf{R}_{\text {int }}^{d} \\
\mathbf{R}_{\text {int }}^{\mathfrak{d}} \\
\mathbf{R}_{\text {int }}^{\varsigma}
\end{array}\right]
$$

Finally, the enhancing strains are eliminated from the previous system by means of a standard condensation procedure yielding:

$$
\left[\begin{array}{ll}
\mathbf{k}_{d d}^{*} & \mathbf{k}_{d \mathfrak{d}}^{*} \\
\mathbf{k}_{\mathfrak{d} d}^{*} & \mathbf{k}_{\mathfrak{d} \mathfrak{d}}^{*}
\end{array}\right]\left[\begin{array}{c}
\Delta \mathbf{d} \\
\Delta \overline{\mathfrak{d}}
\end{array}\right]=\left[\begin{array}{c}
\mathbf{R}_{\text {ext }}^{d} \\
\mathbf{0}
\end{array}\right]-\left[\begin{array}{c}
\mathbf{R}_{\mathrm{int}}^{d *} \\
\mathbf{R}_{\mathrm{int}}^{\mathfrak{d} *}
\end{array}\right]
$$

where the modified residuals and tangent matrices are given as:

$$
\begin{gathered}
\mathbf{k}_{\mathrm{dd}}^{*}=\mathbf{k}_{d d}-\mathbf{k}_{d \varsigma} \mathbf{k}_{\varsigma \varsigma}^{-1} \mathbf{k}_{\varsigma d}, \quad \mathbf{k}_{d \mathfrak{d}}^{*}=\mathbf{k}_{d \mathfrak{d}}-\mathbf{k}_{d \varsigma} \mathbf{k}_{\varsigma \varsigma}^{-1} \mathbf{k}_{\varsigma \mathfrak{d}}, \\
\mathbf{k}_{\mathfrak{d} d}^{*}=\mathbf{k}_{\mathfrak{d} d}-\mathbf{k}_{\mathfrak{d} \varsigma} \mathbf{k}_{\varsigma \varsigma}^{-1} \mathbf{k}_{\varsigma d}, \quad \mathbf{k}_{\mathfrak{d} \mathfrak{d}}^{*}=\mathbf{k}_{\mathfrak{d} \mathfrak{d}}-\mathbf{k}_{\mathfrak{d} \varsigma} \mathbf{k}_{\varsigma \varsigma}^{-1} \mathbf{k}_{\varsigma \mathfrak{d}}, \\
\mathbf{R}_{\mathrm{int}}^{d *}=\mathbf{R}_{\mathrm{int}}^{d}-\mathbf{k}_{d \varsigma} \mathbf{k}_{\varsigma \varsigma}^{-1} \mathbf{R}_{\mathrm{int}}^{\varsigma}, \quad \mathbf{R}_{\mathrm{int}}^{\mathfrak{d} *}=\mathbf{R}_{\mathrm{int}}^{\mathfrak{d}}-\mathbf{k}_{\mathfrak{d} \varsigma} \mathbf{k}_{\varsigma \varsigma}^{-1} \mathbf{R}_{\mathrm{int}}^{\varsigma} .
\end{gathered}
$$

The detailed derivation of the previous formulation is rigourously addressed in [39], whose particular aspects are omitted here for the sake of conciseness.

Finally, from a constitutive point of view, the present solid shell approach endows the combination of the equivalent single layer (ESL) and approach layer-wise (LW) approaches to model the mechanical response of thin and moderately thick composite laminates in an efficient and robust manner [50]. 


\section{Results}

To validate the proposed modeling strategies, the predictions of the coupled FFMs model and the predictions of the PF approach of brittle fracture are compared with experimental results obtained from open-hole tensile tests carried out on different thin-ply composite systems.

\subsection{Finite Fracture Mechanics results}

In [6], the notched response of two quasi-isotropic non-crimp fabric thin-ply laminates produced from a T700/AR-2527 carbon fiber-reinforced epoxy system was studied based on a thorough experimental test campaign. Specific details of the experimental program are omitted here for the sake of conciseness. Thin-ply bi-angle non-crimp fabrics with an areal weight of $150 \mathrm{~g} / \mathrm{m}^{2}$ (or $75 \mathrm{~g} / \mathrm{m}^{2}$ per unidirectional ply) were selected. The laminates were $2.0 \mathrm{~mm}$ and $1.3 \mathrm{~mm}$ thick. In the first (laminate 1), a fully dispersed stacking sequence was adopted. In the second (laminate 2), some ply clustering was obtained by placing two unidirectional plies, from different non-crimp fabric bi-angle layers, with the same fiber orientation adjacent to each other.

The tests included an open-hole size effect study for specimens loaded in tension [6]. Table 1 shows the material properties needed by the proposed FFMs modeling strategy, which are also used for the PF method, see Section 3.2.

Table 1: Laminates mechanical properties.

\begin{tabular}{lllllll}
\hline $\begin{array}{l}\text { Laminate } \\
\text { system }\end{array}$ & $\begin{array}{l}E_{x} \\
(\mathrm{MPa})\end{array}$ & $\begin{array}{l}E_{y} \\
(\mathrm{MPa})\end{array}$ & $\begin{array}{l}G_{x y} \\
(\mathrm{MPa})\end{array}$ & $\begin{array}{l}\nu_{x y} \\
(-)\end{array}$ & $\begin{array}{l}X_{T}^{L} \\
(\mathrm{MPa})\end{array}$ & $\begin{array}{l}\mathcal{G}_{I c} \\
(\mathrm{~N} / \mathrm{mm})\end{array}$ \\
\hline T700/AR-2527 & & & & & & \\
laminate 1 & 42572 & 42572 & 16306 & 0.305 & 800 & 34.3 \\
laminate 2 & 42572 & 42572 & 16306 & 0.305 & 710 & 33.6 \\
M40JB/ThinPreg $\mathbf{8 0 E P / C F}$ & 80197 & 80197 & 30494 & 0.315 & 814 & 11.7 \\
\hline
\end{tabular}

Based on the laminate characteristics, homogenized laminate mechanical properties are considered. Therefore, the laminate elastic, strength and fracture properties are directly used to obtain the notched strength predictions. It is noted that, in both cases, only independently measured laminate properties are used; empirical factors or calibration parameters are not required.

Table 2 shows the average open-hole tensile strengths for both laminates and for each specimen geometry, here presented for different hole diameters $(2 R, R$ denoting the radius of the hole). In addition to the previous non-crimp fabric laminates, a similar open-hole analysis was conducted in $[9,55]$ on a $2.4 \mathrm{~mm}$ thick quasi-isotropic thin ply laminate produced from M40JB/ThinPreg 80EP/CF carbon fiber-reinforced epoxy unidirectional tape layers, whose results are also used in the current study. Specifically, Furtado et al. [55] showed a clear size effect on the notched strength results on a laminate where each unidirectional ply had an areal weight of only $30 \mathrm{~g} / \mathrm{m}^{2}$. 
Table 2: Open-hole tensile strength test results and comparison with the FFMs and PF model predictions.

\begin{tabular}{|c|c|c|c|c|c|c|}
\hline \multirow{2}{*}{\multicolumn{2}{|c|}{$\begin{array}{l}\text { Composite system and } \\
\text { specimen geometry }\end{array}$}} & \multirow{2}{*}{$\begin{array}{l}\text { Experimental } \\
\text { results }\end{array}$} & \multicolumn{2}{|c|}{ Predictions } & \multicolumn{2}{|c|}{ Relative error } \\
\hline & & & FFMs & $\mathrm{PF}$ & FFMs & PF \\
\hline \multicolumn{7}{|c|}{ T700/AR-2527 - laminate 1} \\
\hline$W /(2 R)=4$ & $2 R=3 \mathrm{~mm}$ & $432 \mathrm{MPa}$ & $465 \mathrm{MPa}$ & $415 \mathrm{MPa}$ & $7.8 \%$ & $-3.9 \%$ \\
\hline$W /(2 R)=4$ & $2 R=6 \mathrm{~mm}$ & $385 \mathrm{MPa}$ & $392 \mathrm{MPa}$ & $368 \mathrm{MPa}$ & $1.9 \%$ & $-4.4 \%$ \\
\hline$W /(2 R)=4$ & $2 R=10 \mathrm{~mm}$ & $367 \mathrm{MPa}$ & $342 \mathrm{MPa}$ & $361 \mathrm{MPa}$ & $-5.8 \%$ & $-1.6 \%$ \\
\hline \multicolumn{7}{|c|}{ T700/AR-2527 - laminate 2} \\
\hline$W /(2 R)=4$ & $2 R=3 \mathrm{~mm}$ & $448 \mathrm{MPa}$ & $447 \mathrm{MPa}$ & $403 \mathrm{MPa}$ & $-0.3 \%$ & $-10.4 \%$ \\
\hline$W /(2 R)=4$ & $2 R=6 \mathrm{~mm}$ & $390 \mathrm{MPa}$ & $385 \mathrm{MPa}$ & $358 \mathrm{MPa}$ & $-1.2 \%$ & $-8.2 \%$ \\
\hline$W /(2 R)=4$ & $2 R=10 \mathrm{~mm}$ & $380 \mathrm{MPa}$ & $338 \mathrm{MPa}$ & $345 \mathrm{MPa}$ & $-11.1 \%$ & $-9.2 \%$ \\
\hline \multicolumn{7}{|c|}{ M40JB/ThinPreg 80EP/CF } \\
\hline$W /(2 R)=6$ & $2 R=1 \mathrm{~mm}$ & $551 \mathrm{MPa}$ & $559 \mathrm{MPa}$ & $524 \mathrm{MPa}$ & $1.5 \%$ & $-6.3 \%$ \\
\hline$W /(2 R)=6$ & $2 R=2 \mathrm{~mm}$ & $470 \mathrm{MPa}$ & $475 \mathrm{MPa}$ & $447 \mathrm{MPa}$ & $1.0 \%$ & $-5.9 \%$ \\
\hline$W /(2 R)=6$ & $2 R=6 \mathrm{~mm}$ & $365 \mathrm{MPa}$ & $356 \mathrm{MPa}$ & $351 \mathrm{MPa}$ & $-2.5 \%$ & $-3.8 \%$ \\
\hline
\end{tabular}

Analyzing the data reported in Table 2, it can be seen that the notched strength predictions of the FFMs model have a maximum relative error of approximately 11\%. This larger discrepancy is obtained for the T700/AR-2527 carbon/epoxy laminate with ply clustering (laminate 2). For laminates with very thin plies, as in the M40JB/ThinPreg 80EP/CF laminate, with an individual ply thickness of only $0.03 \mathrm{~mm}$, the predictions differ from the experimental results by less than $3 \%$. This shows the ability of the coupled FFMs criterion to accurately predict failure of laminates exhibiting an intralaminar fiber-dominated netsection failure mode $[7,23,40,56]$. In these cases, particularly in laminates with very thin plies, the assumptions of the FFMs model regarding negligible subcritical damage growth, such as matrix transverse and split cracking and delamination, are perfectly applicable. This means that damage growth can be lumped into a fracture process zone, of finite size, confined to the notch boundaries, and that an analytical representation of the fracture phenomenon is able to accurately represent failure of the notched plate, as long as the boundary value problem can also be solved analytically [7, 23, 40] (or semi-analytically [56]) with a tractable mathematical representation.

Regarding the failure characteristics, based on the experimental evidences, it can be stated that both thin ply composite systems exhibited a brittle type of net-section failure mode, with a fracture plane perpendicular to the applied load $[6,9,55]$. Subcritical damage such as dispersed transverse cracking or delamination was absent. Some pull-out failures were observed in laminate 2 of the T700/AR-2527 composite, originating more irregular fracture surfaces [6]. Nevertheless, based on full-field experimental analysis (viz. digital image correlation) $[6,55]$, it was observed, in both composite systems, that intralaminar damage propagates from the hole edge towards the straight free edges in a plane perpendicular to the loading direction, and 
never parallel to the fibers on the off-axis or longitudinal plies. Because subcritical damage mechanisms such as matrix cracking and delamination are precluded, unstable fracture propagates soon after an intralaminar fracture process zone has formed in the vicinity of the open hole. This means that the assumptions of brittle fracture of the analytical FFMs model are valid for this class of composite materials. Similarly, according to these evidences, the failure and strength analysis of thin ply laminates can be also tackled using novel PF methods.

\subsection{Phase Field results}

As discussed in Section 3.1, the use of the coupled energy-stress FFMs method allows straightforward and accurate strength predictions for simple loading conditions and geometries of thin ply composite laminates to be accomplished. However, in the case of more complex applications, also at industrial level, a computational approach is required in order to provide a robust methodology which can also trigger the failure development upon failure.

Among the different existing numerical methods based on FEM, PF models represent a plausible modeling option for thin ply laminates due to their brittle character upon failure [31, 32]. Specifically, standard continuum damage models tend to fail capturing very brittle behavior due to the abrupt change in the specimen stiffness, whereas enriched-based approaches (XFEM and EFEM) require complex crack tracking algorithms to be additionally coded. These limitations can be overcome using the recently proposed PF models of brittle fracture.

As a consequence of the slender character of thin ply laminates, the current PF model relies on the enhanced assumed solid shell version developed in [39]. This method has been successfully implemented into the purpose FE codes ABAQUS and FEAP, whose specific details regarding the numerical procedure are omitted here for the sake of brevity. In addition, based on the experimental data, the present study is restricted to geometrically linear analysis. Thus, from the mechanical point of view, a Kirchhoff-SaintVenant constitutive formulation is adopted using a homogenized material formulation at laminate level. The tangent material tensor can be formally obtained as: $\mathbb{C}=\partial_{\varepsilon \varepsilon} \Psi(\varepsilon)$, with $\Psi(\varepsilon)$ representing the Helmholtz free energy function. In the convective curvilinear setting this operator reads:

$$
\mathbb{C}=\mathbb{C}^{i j k l} \mathbf{G}_{i} \otimes \mathbf{G}_{j} \otimes \mathbf{G}_{k} \otimes \mathbf{G}_{l},
$$

where $\mathbb{C}^{i j k l}$ are the contravariant components.

In line with $[32,33]$, the following positive-negative decomposition of the free energy density is also 
assumed:

$$
\begin{aligned}
\psi(\varepsilon, \mathfrak{d}) & =\mathfrak{g}(\mathfrak{d}) \psi_{+}^{e}(\boldsymbol{\varepsilon})+\psi_{-}^{e}(\boldsymbol{\varepsilon}), \\
\psi_{+}^{e}(\varepsilon) & =\frac{\lambda}{2}\left(\langle\operatorname{tr}[\varepsilon]\rangle_{+}\right)^{2}+\mu \operatorname{tr}\left[\varepsilon_{+}^{2}\right], \\
\psi_{-}^{e}(\varepsilon) & =\frac{\lambda}{2}\left(\langle\operatorname{tr}[\varepsilon]\rangle_{-}\right)^{2}+\mu \operatorname{tr}\left[\varepsilon_{-}^{2}\right] .
\end{aligned}
$$

In the previous expressions, $\varepsilon$ identifies the small strain tensor, $\operatorname{tr}[\bullet]$ is the trace operator, and $\varepsilon=\varepsilon_{+}+\varepsilon_{-}$ stand for the positive and negative parts of $\varepsilon$. The spectral decomposition of the positive part of the strain tensor is given by: $\boldsymbol{\varepsilon}_{+}=\sum_{i=1}^{n_{\text {dim }}}\left\langle\varepsilon^{i}\right\rangle_{+} \mathbf{n}_{\varepsilon}^{i} \otimes \mathbf{n}_{\varepsilon}^{i}$, where $\varepsilon^{i}$ and $\mathbf{n}_{\varepsilon}^{i}$ are the eigenvalues and the eigenvectors of the strain tensor and $\langle\bullet\rangle_{+}=(\bullet+|\bullet|) / 2$.

First, the current PF formulation is verified using two standard applications regarding fracture modes I and II. These benchmark problems consist of a squared plate of unit lateral size $2 L=1 \mathrm{~mm}$ and thickness $t=0.1 \mathrm{~mm}$, with an initial sharp notch of length $L$ placed in the middle of the plate, which is subjected to: (i) uniform vertical displacements $\Delta$ to model mode I fracture (Fig. 3.a), and (ii) horizontal displacement to simulate mode II fracture (Fig. 3.b). The displacements at the opposite side of the plate are fully restrained. Reproducing [32], the Lamé constants are $\lambda=121.15 \times 10^{3} \mathrm{~N} / \mathrm{mm}^{2}, \mu=80.77 \times 10^{3} \mathrm{~N} / \mathrm{mm}^{2}$, whereas the phase field parameters are equal to $\mathcal{G}_{c}=2.7 \mathrm{~N} / \mathrm{mm}$ and $l=0.015 \mathrm{~mm}$. The simulations are conducted using 15000 elements for the discretization of the plate with localized refinements around the region of the potential crack development. The contour plot of the phase field variable of the ABAQUS and FEAP versions are depicted on the right side of Figs. 3.a and 3.b, corresponding to the fracture modes I and II, respectively. The resulting crack path satisfactorily agrees with previous investigations, see $[32,33]$ for further details.

Subsequently, the proposed PF model is validated through the consideration of the test cases discussed in Section 3.1. The current study employs equivalent homogenized laminate material properties [53], which are coincident with those reported in Table 1, and setting the regularization parameter for the PF model $l=0.2 \mathrm{~mm}$. This value for $l$ is chosen in such a way that at the final imposed displacement (replicating the experimental data), the simulations trigger fully degraded states around the stress concentrator. It is worth mentioning that one of the main characteristics of the PF approach is the fact that it precludes the use of any specific stress-based strength criterion for damage onset and growth, since it is based on the direct competition between the strain and fracture energy terms. However, the length scale parameter $l$ has a strong influence on the strength prediction as discussed in [57], being still an open question of notable importance in practice.

The simulations concern different open-hole specimens subjected to uniform loading upon failure. The prescribed boundary conditions are set coincident with those depicted in Fig. 1 in order to reproduce the experimental conditions. The plates are discretized using approximately 7000 solid shell elements with standard mesh refinement around the hole, where failure events are expected to take place. The principal 
a
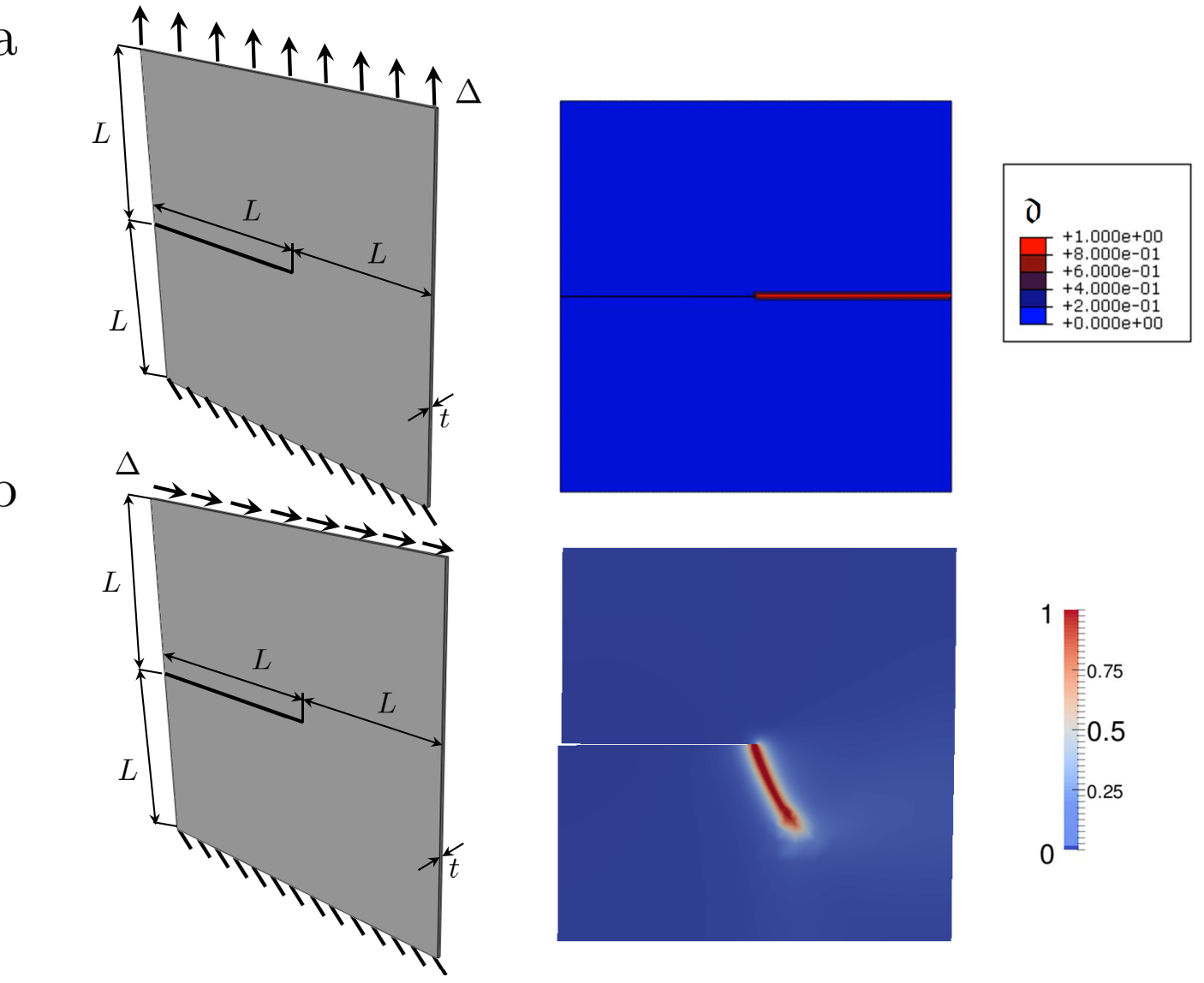

Figure 3: Verification of the PF model of brittle fracture through in-plane fracture tests. (a) Fracture mode I: geometry and contour plot of phase field variable (ABAQUS implementation). (b) Fracture mode II: geometry and contour plot of phase field variable (FEAP implementation).

constraint with respect of the mesh size is the fact that at least two elements within $l$ are needed to obtained a reliable regularization [58].

Table 2 shows the comparison between the numerical simulations using the PF method and the experimental data. Analyzing these results, a good agreement between the maximum stress predictions and the experimental results can be observed, with slight deviations within the range 10.4-1.6\%. For laminates with very thin plies, corresponding to M40JB/ThinPreg $80 \mathrm{EP} / \mathrm{CF}$, these deviations are lower than $7 \%$. It is also worth mentioning that, differing from the FFMs approach, the PF model uniformly underestimate the maximum strength of each specimen. These data corroborates the ability of the PF for accurate failure prediction of open-hole specimens under tensile loading with similar level of agreement with respect to the experimental data and FFMs, and capturing the well-known size effects.

It is important to note that, in addition to the ultimate strength estimation, the current numerical method is able to predict the plate stiffness evolution along the loading process. In this concern, for 
example, Fig. 4 shows the results corresponding to the predicted remote stress-displacement evolution curve (dashed line) for the specimens T700/AR-2527 - laminate 1 and the experimental data (solid lines). In this graph, the experimental data features a quasi-perfect linear evolution of the response up to the sudden failure around the maximum load. From a qualitative point of view, the predictions using the PF method satisfactorily agree with the experimental measures previously reported in [6], suggesting again that the failure is dominated by fiber breakage. Note however that slight discrepancies of the numerical estimations (dashed line) regarding the specimen stiffness with respect to the actual load-displacement evolution curve (solid lines) are obtained. These deviations can be attributed to the adoption of a homogenized constitutive model at laminate level. However, based on the data herein reported, it can be concluded that this simple model leads to the achievement of a good compromise between reliability and numerical efficiency. A similar level of accuracy is also obtained for the remaining open-hole plates under analysis in the current investigation, though their corresponding results are omitted for brevity reasons.
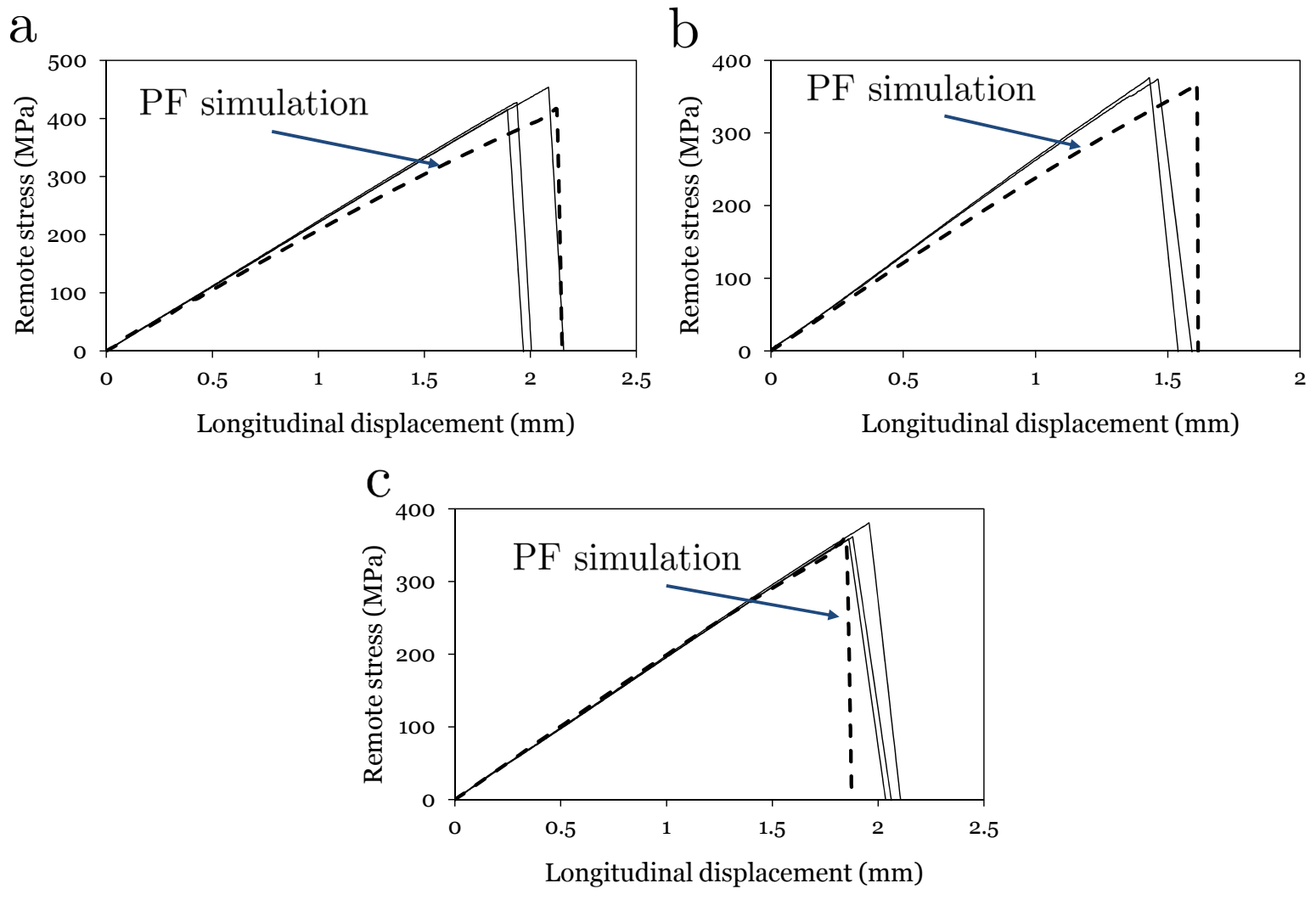

Figure 4: Experimental-numerical correlation of the remote stress-displacement evolution for the specimens T700/AR-2527 laminate 1. (a) $W /(2 R)=4$ and $2 R=3 \mathrm{~mm}$. Experimental results correspond to solid lines, whereas numerical data are represented by dashed lines. (b) $W /(2 R)=4$ and $2 R=6 \mathrm{~mm}$. (c) $W /(2 R)=4$ and $2 R=10 \mathrm{~mm}$. 
The crack pattern corresponding to the specimen T700/AR-2527 - laminate 1 complying with $W /(2 R)=$ 4 and $2 R=10 \mathrm{~mm}$ is depicted in Fig. 5.a at different stages along the loading process. This graph shows a brittle type of net-section failure mode that is developed perpendicular to the direction of the applied load. As expected, the predicted crack pattern is concentrated around the notches and evolves symmetrically till reaching the extremes of the plate. These failure evolutions reproduce with high accuracy the experimental observations after failure [6], see Fig. 5.b, which is identically replicated in the remaining thin ply laminates herein analyzed.

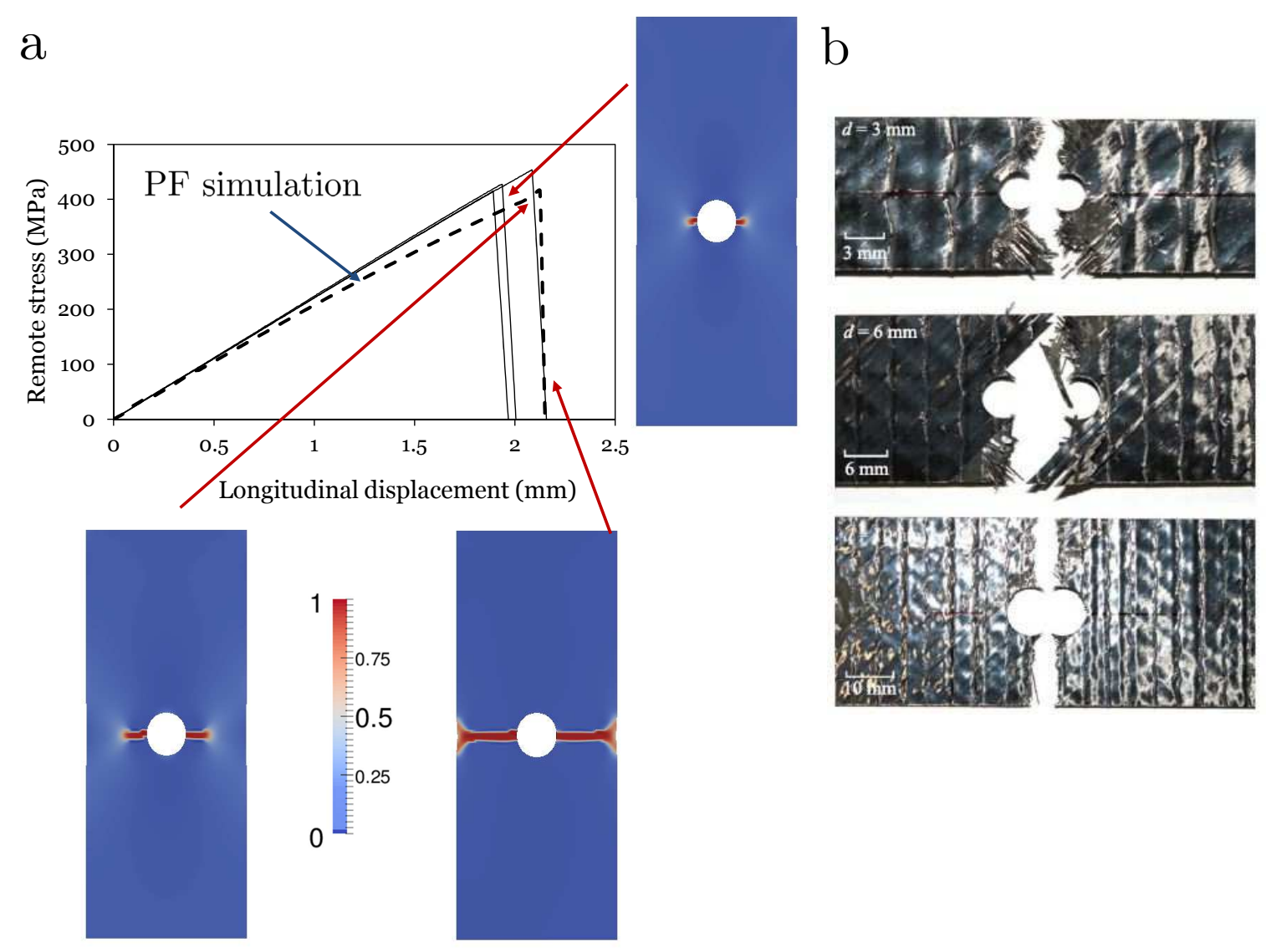

Figure 5: Open-hole results for the T700/AR-2527 - laminate 1 with $W /(2 R)=4$ and $2 R=10 \mathrm{~mm}$. (a) Experimentalnumerical correlation of the remote stress-displacement evolution and crack patterns at different stages. (b) Representative specimens after testing: characteristic failure [6]. Experimental results correspond to solid lines, whereas numerical data are represented by dashed lines.

Therefore, in view of the previous results, it can be stated that the current numerical method relying on the PF approach of brittle fracture is able to accurately predict the notched strength and mechanical behavior of thin ply laminates, capturing their brittle response without the use of any additional remeshing technique, kinematic enrichment and/or crack tracking algorithm. 
Performing a direct comparison between both approaches herein considered, from a qualitative standpoint, it is worth noting that in contrast to FFMs, PF approach of brittle fracture provides valuable information regarding the crack initiation load, growth path and final strength on the specimen. Moreover, this latter method can be employed in a straightforward manner to strength and failure predictions independent of the geometric characteristics of the specimen and the loading conditions, but incurring in high computational efforts. In contrast, FFMs yields to accurate and fast estimations, but confined to simpler geometries and loading/supporting conditions. Finally, from a quantitative perspective, both methodologies provide reliable strength predictions of notched thin ply laminates with a similar level of accuracy with respect to the experimental data, and capturing the well-known size effects. In particular, the underestimation of the strength estimations using the PF method might be related to the role of the length scale $l$. A rigourous, investigation with regard to the role of this parameter and the possible complementarity between both approaches is beyond the scope of the present study.

\section{Concluding remarks}

In this paper, accurate failure and strength analysis of open-hole thin ply laminates has been performed using two methods: (i) the analytical coupled energy-stress Finite Fracture Mechanics (FFMs) technique, and (ii) the FE-based Phase Field (PF) approach of brittle fracture complying with a multi-field variational formulation.

On the theoretical side, the coupled energy-stress FFMs approach has been proven to provide accurate strength predictions of open-hole thin ply laminates, capturing the well-known size effects with great accuracy. Because it is based on analytical (or semi-analytical) solutions, fast strength predictions can be obtained, without requiring fitting parameters or calibration factors, but only independently measured material properties: the laminate strength and fracture toughness. Therefore, the FFMs model provides an accurate, fast and inexpensive tool suitable for preliminary design and optimization of thin ply composite structures.

On the computational side, the recently proposed PF method to simulate brittle fracture in solids has been successfully applied. One of the principal aspects of this nonlocal damage approach concerns the derivation of the regularized crack surface functional, which converges to the sharp crack topology for vanishing crack regularization parameter $l$. The proposed PF model has been incorporated into an enhanced assumed solid shell element, which precluded the deterioration of the solution using the Enhanced Assumed Strain (EAS) method. This modeling tool allowed: (1) the reliable prediction of the ultimate strength of the plates, and (2) capturing the plate stiffness evolution along the loading process and the crack patterns within the specimen. The results obtained were correlated with the experimental data, showing a satisfactory level of accuracy similar to FFMs. Therefore, the current PF method provides reliable qualitative and quantitative 
comparisons with experimental data, confirming its accuracy and applicability to simulate fracture events in thin ply laminates at industrial level in an efficient manner.

\section{Acknowledgments}

JR is grateful to the support of the research projects funded by the Spanish Ministry of Economy and Competitiveness/FEDER (Projects DPI2012-37187, MAT2015-71036-P and MAT2015-71309-P) and the Andalusian Government (Projects of Excellence No. P11-TEP-7093 and P12-TEP-1050). AE would like to thank the financial support provided by FCT - Fundação para a Ciência e a Tecnologia through National Funds in the scope of project MITP-TB/PFM/0005/2013.PP Camanho gratefully acknowledges the funding of Project NORTE-01-0145-FEDER-000022 - SciTech - Science and Technology for Competitive and Sustainable Industries, co-financed by Programa Operacional Regional do Norte (NORTE2020), Fundo Europeu de Desenvolvimento Regional (FEDER). MP and JR gratefully acknowledge financial support of the European Research Council (ERC), Grant No. 306622 through the ERC Starting Grant "Multi-field and multi-scale Computational Approach to Design and Durability of PhotoVoltaic Modules" - CA2PVM.

\section{References}

[1] Tao, J., Sun, C.T. (1998) Influence of ply orientation on delamination in composite laminates. Journal of Composite Materials 32(21):1933-1947.

[2] Dvorak, G.J., Laws, N. (1987) Analysis of progressive matrix cracking in composite laminates II. First ply failure. Journal of Composite Materials 21:309-329.

[3] Camanho, P.P., Dávila, C.G., Pinho, S.T., Iannucci, L., Robinson, P. (2006) Prediction of in situ strengths and matrix cracking in composites under transverse tension and in-plane shear. Composites Part A: Applied Science and Manufacturing $37: 165-176$.

[4] Sihn, S., Kim, R.Y., Kawabe, K., Tsai, S.W. (2007) Experimental studies of thin-ply laminated composites. Composites Science and Technology 67:996-1008.

[5] Saito, H., Takeuchi, H., Kimpara, I. (2012) Experimental evaluation of the damage growth restraining in $90^{\circ}$ layer of thin-ply CFRP cross-ply laminates. Advanced Composite Materials 21:57-66.

[6] Arteiro, A., Catalanotti, G., Xavier, J., Camanho P.P. (2013) Notched response of non-crimp fabric thin-ply laminates. Composites Science and Technology 79:97-114.

[7] Arteiro, A., Catalanotti, G., Xavier, J., Camanho, P.P. (2014) Large damage capability of non-crimp fabric thin-ply laminates. Composites Part A: Applied Science and Manufacturing 63:110-122.

[8] Guillamet, G., Turon, A., Costa, J., Renart, J., Linde P., Mayugo, J.A. (2014) Damage occurrence at edges of non-crimpfabric thin-ply laminates under off-axis uniaxial loading. Composites Science and Technology 98:44-50.

[9] Amacher, R., Cugnoni J., Botsis, J., Sorensen, L., Smith, W., Dransfeld, C. (2014) Thin ply composites: Experimental characterization and modeling of size-effects. Composites Science and Technology 101:121-132.

[10] Fuller, J., Wisnom, M.R. (2015) Pseudo-ductility and damage suppression in thin ply CFRP angle-ply laminates. Composites Part A: Applied Science and Manufacturing 69:64-71.

[11] Ladevèze, P., Lubineau, G. (2001) On a damage mesomodel for laminates: micro-meso relationships, possibilities and limits. Composites Science and Technology 61:2149-2158. 
[12] Camanho, P.P., Maimí, P., Dávila, C.G. (2007) Prediction of size effects in notched laminates using continuum damage mechanics. Composites Science and Technology 67:2715-2727.

[13] Maimí, P., Camanho, P.P., Mayugo, J.A., Dávila, C.G. (2007) A continuum damage model for composite laminates: Part I - Constitutive model. Mechanics of Materials 39:897-908.

[14] Maimí, P., Camanho, P.P., Mayugo, J.A., Dávila, C.G. (2007) A continuum damage model for composite laminates: Part II - Computational implementation and validation. Mechanics of Materials 39:909-919.

[15] Abisset, E., Daghia, F., Ladevèze, P. (2011) On the validation of a damage mesomodel for laminated composites by means of open-hole tensile tests on quasi-isotropic laminates. Composites Part A: Applied Science and Manufacturing 42:1515-1524.

[16] Laurin, F., Carrere, N., Huchette, C., Maire, J.-F. (2013) A multiscale hybrid approach for damage and final failure predictions of composite structures. Journal of Composite Materials 47(20-21):2713-2747.

[17] Camanho, P.P., Bessa, M.A., Catalanotti, G., Vogler, M., Rolfes, R. (2013) Modeling the inelastic deformation and fracture of polymer composites — Part II: Smeared crack model. Mechanics of Materials 59:36-49.

[18] Vogler, M., Rolfes, R., Camanho, P.P. (2013) Modeling the inelastic deformation and fracture of polymer composites — Part I: Plasticity model. Mechanics of Materials 59:50-64.

[19] Leguillon, D. (2002) Strength or toughness? A criterion for crack onset at a notch. European Journal of Mechanics A/Solids 21, 61-72.

[20] Mantič, V. (2009) Interface crack onset at a circular cylindrical inclusion under a remote transverse tension. Application of a coupled stress and energy criterion. International journal of Solids and Structures 46(6):1287-1304.

[21] García IG, Mantič, V. Blázquez A, París F (2014) Transverse crack onset and growth in cross-ply laminates under tension: application of a coupled stress and energy criterion. International journal of Solids and Structures 51:3844-3856.

[22] García IG, Paggi, M., Mantič, V. (2014) Fiber-size effects on the onset of fiber-matrix debonding under transverse tension: a comparison between cohesive zone and finite fracture mechanics models. Engineering Fracture Mechanics 115:96-110.

[23] Camanho, P.P., Erçin, G.H., Catalanotti, G., Mahdi, S., Linde, P. (2012) A finite fracture mechanics model for the prediction of the open-hole strength of composite laminates. Composites Part A: Applied Science and Manufacturing 43(8):1219-1225.

[24] Martin, E., Leguillon, D., Carrère, N. (2012) A coupled strength and toughness criterion for the prediction of the open hole tensile strength of a composite plate. International Journal of Solids and Structures 49:3915-3922.

[25] J. Lemaitre, R. Desmorat (2005) Engineering Damage Mechanics: Ductile, Creep, Fatigue and Brittle Failures, SpringerVerlag, Berlin.

[26] N. Moës, J. Dolbow, T. Belytschko (1999) A finite element method for crack growth without remeshing, Int. J. Numer. Methods Engrg. 46(1):131-150.

[27] Linder, C., Armero, F. (2007) Finite elements with embedded strong discontinuities for the modeling of failure in solids, Int. J. Numer. Methods Engrg. 72:1391-1433.

[28] Diyaroglu, C., Oterkus, E., Madenci, E., Rabczuk, T., Siddiq, A. (2016) Peridynamic modeling of composite laminates under explosive loading. Composite Structures 144:14-23.

[29] Feyel F. (2003) A multilevel finite element method ( $\left.\mathrm{FE}^{2}\right)$ to describe the response of highly non-linear structures using generalized continua. Computer Methods in Applied Mechanics and Engineering, 192(28-30):3233-3244.

[30] Budarapu, P.R., Gracie, R., Bordas, S.P.A., Rabczuk, T. (2014) An adaptive multiscale method for quasi-static crack growth, Computational Mechanics 53(6):1129-1148.

[31] Bourdin, B., Francfort, G.A., Marigo, J.J. (2008) The variational approach to fracture. J. Elast. 91(1-3):5-148.

[32] Miehe, C., Hofacker, M., Welschinger, F. (2010) A phase field model for rateindependent crack propagation: robust algorithmic implementation based on operator splits. Comput. Methods Appl. Mech. Engrg. 199(45-48):2765-2778. 
[33] Miehe, C., Welschinger, F., Hofacker, M. (2010) Thermodynamically consistent phase field models of fracture: variational principles and multi-field fe-implementations. International Journal for Numerical Methods in Engineering, 83(10):12731311.

[34] Areias, P.M.A., Belytschko, T. (2005) Non-linear analysis of shells with arbitrary evolving cracks using XFEM. International International Journal for Numerical Methods in Engineering, 62:384-415.

[35] Rabczuk, T., Areias P.M.A. (2006) A meshfree thin shell for arbitrary evolving cracks based on an extrinsic basis. CMESComputer Modeling in Engineering and Sciences, 16:115-130.

[36] Rabczuk, T., Areias, P.M.A., Belytschko, T. (2007) A meshfree thin shell method for nonlinear dynamic fracture. International International Journal for Numerical Methods in Engineering, 72(5):524-548.

[37] Amiri, F., Millán D., Shen, Y., Rabczuk, T., Arroyo M. (2014) Phase-field modeling of fracture in linear thin shells. Theoretical and Applied Fracture Mechanics 69:102-109.

[38] Budarapu, P.R., Reinoso, J., Paggi, M. (2017) Concurrently coupled solid shell based adaptive multiscale method for fracture. Computer Methods in Applied Mechanics and Engineering 319, 338-365.

[39] Reinoso, J., Paggi, M., Linder, C. (2017) Phase field modeling of brittle fracture for enhanced assumed strain shells at large deformations: formulation and finite element implementation. Computational Mechanics 59(6):981-1001.

[40] Arteiro, A., Catalanotti, G., Xavier, J., Camanho, P.P. (2013) Notched response of non-crimp fabric thin-ply laminates: Analysis methods. Composites Science and Technology 88:165-171.

[41] Tan, S.C. (1994) Stress Concentrations in Laminated Composites. Lancaster: Technomic.

[42] Newman Jr, J.C. (1983) A nonlinear fracture mechanics approach to the growth of small cracks. In: Proceedings of the AGARD conference $328(6)$.

[43] Whitney, J.M., Nuismer, R.J. (1974) Stress fracture criteria for laminated composites containing stress concentrations. Journal of Composite Materials 8:253-265.

[44] Borden, M.J., Verhoosel, C.V., Scott, M.A., Hughes, T.J.R., Landis, C.M. (2012) A phase field description of dynamic brittle fracture. Comput. Methods Appl. Mech. Engrg. 217220:7795.

[45] Hesch, C.,Weinberg, K. (2014) Thermodynamically consistent algorithms for a finite-deformation phase field approach to fracture. Int. J. Numer. Methods Eng. 99(12):906-924.

[46] Zhang, X., Krischok, A., Linder, C. (2016) A variational framework to model diffusion induced large plastic deformation and phase field fracture during initial two-phase lithiation of silicon electrodes. Computer Methods in Applied Mechanics and Engineering, 312:51-77.

[47] Areias, P., Rabczuk, T., Msekh, M.A. (2016) Phase-field analysis of finite-strain plates and shells including element subdivision. Computer Methods in Applied Mechanics and Engineering, 2016.

[48] Hauptmann, R., Schweizerhof, K., Doll, S. (2000) Extension of the solid-shell concept for application to large elastic and large elastoplastic deformations. Int. J. Numer. Methods Eng. 49:1121-1141.

[49] Vu-Quoc, L., Tan, X.G. (2003) Optimal solid shells for non-linear analyses of multilayer composites. I. Statics. Comput. Methods Appl. Mech. Eng. 192:975-1016.

[50] Reinoso, J., Blázquez, A. (2016) Geometrically nonlinear analysis of functionally graded power-based and carbon nanotubes reinforced composites using a fully integrated solid shell element, Composite Structures 152:277-294.

[51] M. Bischoff , E. Ramm (1997) Shear deformable shell elements for large strains and rotations. Int J Numer Methods Eng 40:4427-4449.

[52] Simo, J.C., Armero, F. (1992) Geometrically nonlinear enhanced strain mixed methods and the method of incompatible modes. Int J Numer Methods Eng 33:1413-1449.

[53] Reinoso, J., Blázquez, A. (2016) Application and finite element implementation of 7-parameter shell element for geometrically nonlinear analysis of layered CFRP composites, Composite Structures 139:263-276. 
[54] Klinkel, S., Wagner, W. (1997) A geometrical nonlinear brick element based on the EAS-method. International Journal for Numerical Methods in Engineering 40: 4529-4545.

[55] Furtado, C., Arteiro, A., Catalanotti, G., Xavier, J., Camanho, P.P. (2016) Selective ply-level hybridisation for improved notched response of composite laminates. Composite Structures 145:1-14.

[56] Catalanotti, G., Camanho, P.P. (2013) A semi-analytical method to predict net-tension failure of mechanically fastened joints in composite laminates. Composites Science and Technology 76:69-76.

[57] Nguyen, T.T., Yvonnet, J., Bornert, M., Chateau, C., Sab, K., Romani, R., Le Roy, R. (2016). On the choice of parameters in the phase field method for simulating crack initiation with experimental validation. International Journal of Fracture 197(2):213-226.

[58] Paggi, M., Reinoso, J. Revisiting the problem of a crack impinging on an interface: a modeling framework for the interaction between the phase field approach for brittle fracture and the interface cohesive zone model. Computer Methods in Applied Mechanics and Engineering 321, 145-172. 